\title{
Variação aguda não uniforme da espessura muscular ao longo do peitoral maior em resposta ao supino reto em homens treinados
}

\section{Non-uniform acute variation among pectoralis major's muscle thickness after bench press in trained men}

\section{Variación aguda no uniforme del espesor muscular al largo del pectoral mayor en respuesta al press de banca en hombres entrenados}

\author{
iD (9) Bruno Felipe Mendonça Leitão \\ Universidade Federal do Rio de Janeiro, Rio de Janeiro, Rio de Janeiro, Brasil \\ brunofmleitao@gmail.com \\ iD Liliam Fernandes de Oliveira \\ Universidade Federal do Rio de Janeiro, Rio de Janeiro, Rio de Janeiro, Brasil \\ liliam.oliva@gmail.com \\ (iD) Thiago Torres da Matta \\ Universidade Federal do Rio de Janeiro, Rio de Janeiro, Rio de Janeiro, Brasil \\ ttmatta@eefd.ufrj.br
}

\begin{abstract}
Resumo: O objetivo do estudo foi comparar a variação da espessura muscular (EM) ao longo do peitoral maior (PM) após três séries de 8 a 12 repetições máximas de supino reto. A amostra foi composta por 12 homens treinados. O comportamento da EM foi avaliado pré e imediatamente após a realização do exercício através de ultrassonografias, em três sítios do feixe esternal do PM: medial (próximo ao esterno), central e lateral (próximo ao úmero). Como resultado, o sítio medial apresentou variação relativa da EM de $14,78 \%$, o central de $11,83 \%$ e o lateral de $10,04 \%$, com diferença significativa apenas entre o sítio medial quan-
\end{abstract}


do comparado ao lateral $(\mathrm{p}=0,036)$. Tal efeito pode estar relacionado a maior ativação do trecho medial do PM durante o supino reto ou pela específica morfologia do PM.

Palavras-chave: Arquitetura muscular. Músculo esquelético. Hiperemia. Ultrassonografia. Treinamento de força.

Abstract: The aim of this study was to compare the variation of muscle thickness (MT) over the pectoralis major muscle (PM) after 3 sets of 8 to 12 repetitions of bench press. The sample was consisted of 12 trained men. Ultrasound images were acquired, to analyze the MT behavior, at three sites of the PM's sternal head: medial, central and lateral. The medial site had a MT relative variation of $14.78 \%$, the central one of $11.83 \%$ and the lateral one of $10.04 \%$, with a statistical difference observed only between the medial site when compared to the lateral site $(p=0.036)$; The possible cause of these effects might be related to a possible greater activation of the medial site during the bench press or due to PM's morphology. We conclude that the PM shows heterogeneous MT acute variation.

Keywords: Muscle architecture. Skeletal muscle. Hyperemia.

Ultrasonography. Resistance training.

Resumen: El objetivo del estudio fue comparar la variación de la espesura muscular (EM) a lo largo del pectoral mayor (PM) después de tres series de 8 a 12 repeticiones de press de banca. El comportamiento de la EM fue evaluado pre e inmediatamente después de la realización del ejercicio con ultrasonografías, en tres sitios de la cabeza esternal del PM: medial (cerca del esternón), central y lateral (cerca del húmero). Como resultado, el sitio medial presentó $14,78 \%$ de variación relativa de la EM, el central $11,83 \%$ y el lateral $10,04 \%$, con diferencia significativa apenas entre el sitio medial cuando comparado al lateral $(p=0,036)$. Este efecto puede estar relacionado con la mayor activación del sitio medial del PM durante el press de banca o por la morfología del PM.

Palabras clabe: Arquitectura muscular. Músculo esquelético. Hiperemia. Ultrasonografia. Entrenamiento de resistencia.

Submetido em: 30/08/2018

Aceito em: 24/09/2019 


\section{Introdução}

A arquitetura muscular pode sofrer alterações agudas provenientes de uma sessão de treinamento de força (TF) devido ao maior acúmulo de sangue no músculo imediatamente após o exercício realizado (CSAPO; ALEGRE; BARON, 2011) ou desorganização das estruturas miofibrilares (FRIDÉN; LIEBER, 2001). Logo após uma sessão de TF, o volume sanguíneo é redirecionado para a musculatura agonista ao exercício e consequentemente ativada por fatores mecânicos e metabólicos, ou seja, compressão dos vasos sanguíneos pela contração muscular (CLIFFORD et al., 2006) e aumento da demanda por oxigênio (FLAMM et al., 1990).

Dentre os efeitos agudos do TF convencional intenso, cuja fase concêntrica é máxima e excêntrica submáxima, destaca-se, como variável da arquitetura muscular, o aumento da espessura muscular (EM) (AKAGl et al., 2015), normalmente medida em apenas um sítio muscular. Recentemente, a distribuição desse efeito ao longo do músculo vem sendo estudada com mais frequência para determinar respostas agudas não uniformes que possam apontar para possíveis adaptações crônicas estruturais (WAKAHARA et al., 2012, 2013, 2017). Dessa forma, já foi evidenciada hipertrofia heterogênea ao longo de um músculo ou grupamento muscular, por exemplo, para extensores de joelho (BLAZEVICH et al., 2003; EMA et al., 2013; MATTA et al., 2015; WAKAHARA et al., 2017), flexores de cotovelo (MATTA et al., 2011) e extensores de cotovelo (WAKAHARA et al., 2012, 2013), em estudos crônicos que aplicaram um TF específico.

Nesse sentido, Wakahara e colaboradores $(2012,2013,2017)$ avaliaram o redirecionamento agudo do fluxo sanguíneo induzido pelo exercício através do tempo de relaxação (T2) via ressonância magnética e verificaram que a perfusão sanguínea ocorre de forma heterogênea ao longo do tríceps, para a extensão de cotovelo em decúbito dorsal com o ombro flexionado (WAKAHARA et al., 2012) e para a flexão de ombro com extensão de cotovelo 
Variação aguda não uniforme da espessura muscular ao longo do peitoral ... Bruno Felipe Mendonça Leitão • Liliam Fernandes de Oliveira • Thiago Torres da Matta

(WAKAHARA et al., 2013); e reto femoral, para extensão unilateral de joelho (WAKAHARA et al., 2017). No entanto, Akagi e colaboradores (2015), usando o mesmo protocolo agudo que Wakahara e colaboradores (2012), realizaram uma análise mais direta da variação aguda da arquitetura muscular medindo a EM em diferentes sítios do tríceps braquial pré e pós-exercício. Baseado na média dos valores absolutos mostrados, os indivíduos apresentaram variação aparentemente homogênea da EM ao longo do tríceps braquial.

No campo científico, é possível observar que muito se tem estudado sobre as consequências do TF ao longo de uma musculatura, sendo sua grande maioria voltada aos músculos do membro inferior ou superior, como evidenciado acima. O peitoral maior (PM), como músculo do tronco, apresenta grande interesse científico no que diz respeito ao comportamento da sua arquitetura com a aplicação do TF (ABE et al., 2000; KIKUCHI; NAKAZATO, 2017; OGASAWARA et al., 2012; PRESTES et al., 2017) e de sua atividade mioelétrica (BARNETT; KIPPERS; TURNER, 1995; LAUVER; CAYOT; SCHEUERMANN, 2016). Entretanto, apesar de a última ter verificado diferenças espaciais no PM, seu comportamento estrutural ao longo de seu comprimento ainda não foi avaliado, tanto após uma intervenção de TF aguda quanto crônica.

Portanto, o objetivo do presente estudo é de comparar a variação da EM ao longo do PM, pós-supino reto, em indivíduos treinados. Apesar de ter sido apresentada variação heterogênea da perfusão sanguínea durante exercício (WAKAHARA et al., 2012, 2013, 2017), aparentemente, ao avaliar diretamente a EM, essa variação parece ocorrer de maneira homogênea para o tríceps braquial (AKAGl et al., 2015). Embora o PM e a porção longa do tríceps braquial tenham diferentes características quanto à organização das fibras musculares (PETERSON; RAYAN, 2011) a hipótese do presente estudo é que um dos efeitos agudos que uma sessão de supino reto promove na EM do PM também seja homogêneo ao longo de seu comprimento. 


\section{Materiais e métodos}

\section{Amostra}

A amostra foi composta por 12 homens jovens, treinados e familiarizados com o supino reto $(28,50 \pm 4,17$ anos de idade; 79,00 $\pm 7,83 \mathrm{~kg} ; 173,54 \pm 6,57 \mathrm{~cm}$ de estatura; $25,81 \pm 2,06 \mathrm{de} \mathrm{IMC;} 38,50$ $\pm 2,83$ de distância acromial; $38,50 \pm 1,70 \mathrm{~cm}$ de circunferência do braço direito). A amostra foi selecionada por conveniência e respeitou-se os critérios de inclusão adotados para a realização do teste: a) praticar TF com regularidade há, pelo menos, dois anos; b) realizar uma repetição máxima (RM) de supino reto com pelo menos $125 \%$ da massa corporal como sobrecarga (WEIR; WAGNER; HOUSH, 1994); c) possuir circunferência de braço $\geq 35 \mathrm{~cm}$; d) não relatar lesão musculoesquelética de membros superiores; e) não ter realizado TF em um intervalo de, pelo menos, dois dias anteriores ao teste. Inicialmente, a amostra foi composta por 15 indivíduos, no entanto, três foram descartados por não atenderem ao critério de sobrecarga para o exercício.

Antes da intervenção, todos os voluntários foram instruídos pelos avaliadores quanto ao teste e assinaram um termo de consentimento livre e esclarecido de acordo com o projeto aprovado pelo comitê de ética em pesquisa do Hospital Clementino Fraga Filho (número 44100215.6.0000.5257).

\section{Desenho Experimental}

Os voluntários chegaram ao laboratório e tiveram seus dados antropométricos coletados e, posteriormente, repousaram sobre uma maca para que as ultrassonografias fossem obtidas. Em seguida, eles realizaram o aquecimento e o teste e, então, retornaram a maca para a coleta das medidas pós-treino. 


\section{Intervenção}

O supino reto foi o exercício selecionado para o teste e, para isso, os voluntários foram orientados a permanecer em decúbito dorsal, mantendo as costas apoiadas no banco e os pés em contato com o solo durante toda execução do exercício. A distância entre as mãos na barra, cuja massa era de $10 \mathrm{~kg}$, foi padronizada como o dobro da distância entre os acrômios (OGASAWARA et al., 2012), previamente medida $(38,50 \mathrm{~cm} \pm 2,83)$.

Previamente ao teste, foi realizado um aquecimento específico com o intuito de intensificar a ativação neuromuscular e melhorar o desempenho no exercício (SÁ et al., 2016), que consistiu em uma série de supino reto de 10 repetições com $50 \%$ da sobrecarga do teste. A intervenção consistiu em três séries de supino reto com 8 a 12 repetições máximas (RM). Caso necessário, a sobrecarga era reduzida entre cinco e dez por cento (WILLARDSON; SIMAO; FONTANA, 2012), visando manter o número de repetições dentro da faixa estipulada. Foi adotado um intervalo de dois minutos entre as séries, assim como entre o aquecimento e o teste.

Cada repetição foi iniciada da fase excêntrica, a partir do momento em que os voluntários estivessem com extensão total de cotovelos, desciam a barra tocando o peito, até voltar a estender os cotovelos. Durante a intervenção, os voluntários recebiam estímulos verbais dos avaliadores, assim como auxílios para retirar e recolocar a barra no suporte. A série era finalizada quando o voluntário era incapaz de erguer a barra realizando uma amplitude de movimento completa, caracterizando a falha concêntrica.

\section{Medida de espessura muscular}

As ultrassonografias foram realizadas pelo mesmo examinador, longitudinalmente ao PM, alinhado com a articulação do manúbrio com o esterno (encontrada via ultrassonografia) e o tendão do PM, em três sítios da porção esternal do PM: medial (próximo ao esterno), central e lateral (próximo à axila), demarcados à cane- 
Variação aguda não uniforme da espessura muscular ao longo do peitoral ...

Bruno Felipe Mendonça Leitão • Liliam Fernandes de Oliveira • Thiago Torres da Matta

ta na pele dos voluntários para que o transdutor fosse posicionado sempre no mesmo local (Figura 1). O feixe esternal do PM foi escolhido para ser analisado, pois apresenta maior ativação que o clavicular durante o supino reto (LAUVER; CAYOT; SCHEUERMANN, 2016). As ultrassonografias foram adquiridas em dois momentos: antes da intervenção (pré) e, imediatamente, após a intervenção (pós), duas imagens para cada sítio pré e duas pós, totalizando 12 imagens por voluntário. Os voluntários repousaram em decúbito dorsal por, no mínimo, cinco minutos antes da aquisição das ultrassonografias pré-intervenção para acomodação dos tecidos.

Figura 1: Sítios de aquisição das ultrassonografias do PM.

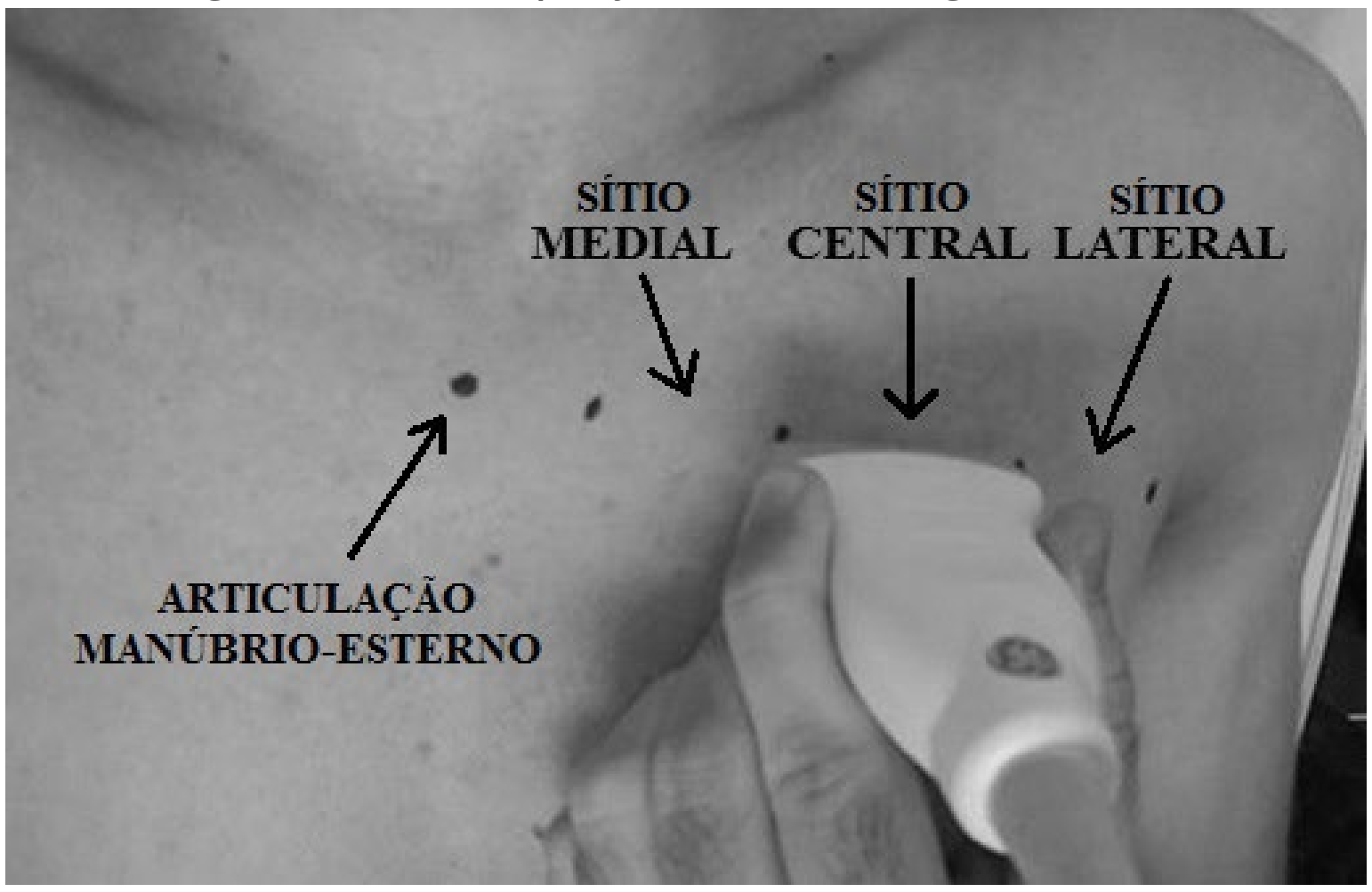

Fonte: Elaborada pelos autores, 2018.

O equipamento utilizado para a aquisição das ultrassonografias foi um aparelho portátil (GE LOGIC e, EUA) com transdutor linear de quatro centímetros. Foi aplicado um gel solúvel em água para um melhor acoplamento acústico do transdutor com a pele. Para aquisição da imagem, foi adotada a profundidade de seis 
Variação aguda não uniforme da espessura muscular ao longo do peitoral ... Bruno Felipe Mendonça Leitão • Liliam Fernandes de Oliveira • Thiago Torres da Matta

centímetros com $8 \mathrm{MHz}$ de frequência excitatória para melhor visualização da aponeurose profunda do PM nas imagens.

O processamento das imagens exportadas foi realizado no programa Imagel (National Institutes of Health, EUA, v.1.43). A EM foi adotada como a distância perpendicular da aponeurose superficial do PM até a aponeurose profunda e foi medida nas duas extremidades (direita e esquerda) e no centro de cada imagem, a média dessas três medidas foi utilizada para análises posteriores (CSAPO; ALEGRE; BARON, 2011) (Figura 2). Com a média de cada imagem, foi determinada a EM média amostral de cada sítio (pré e pós) para cada voluntário, posteriormente, as EM pós foram normalizadas pelas pré do mesmo sítio e para cálculo da variação relativa da EM (EM\%) de cada sítio.

Figura 2: Ultrassonografias dos três sítios do PM, pré e pós-exercício, com as marcações de medição da EM.
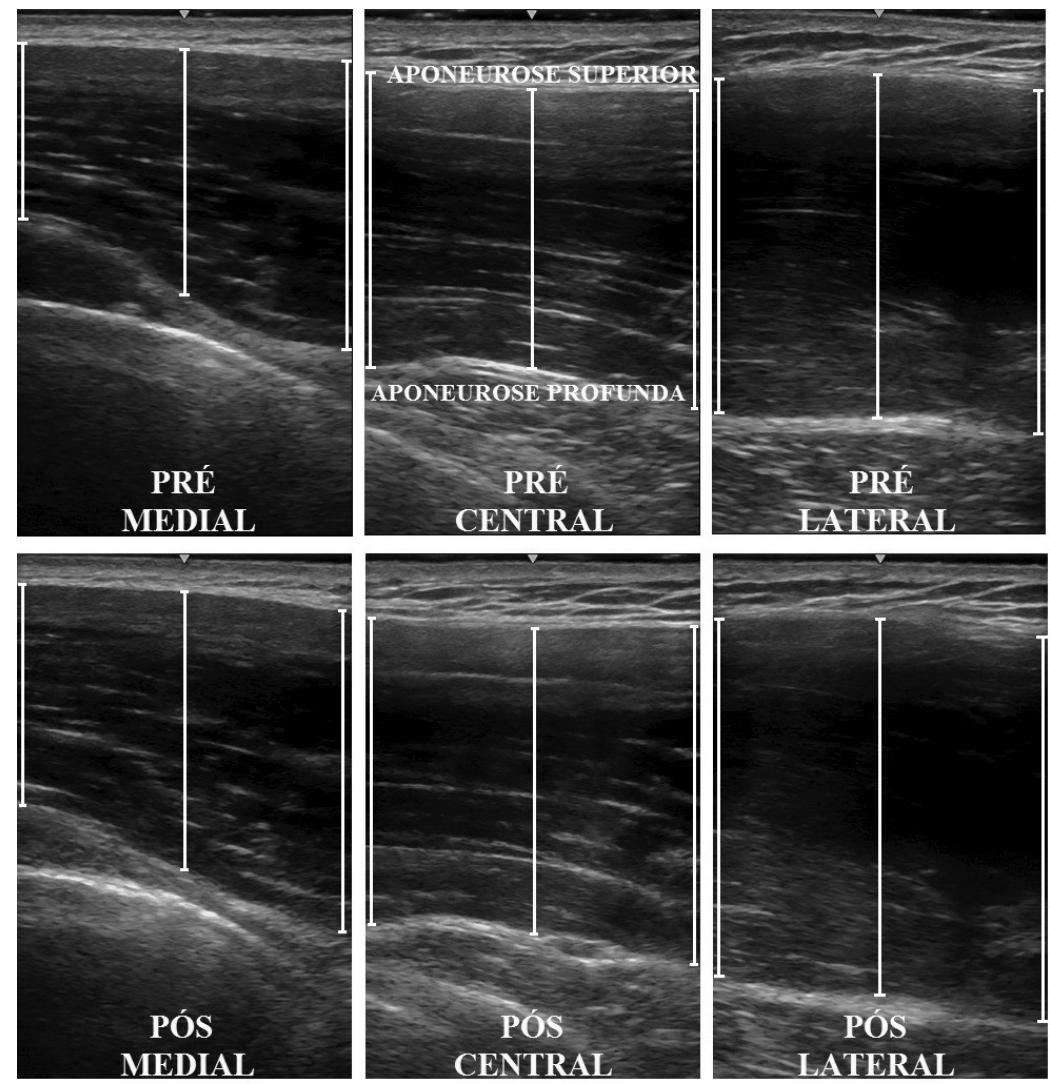

Fonte: Elaborada pelos autores, 2018 
Variação aguda não uniforme da espessura muscular ao longo do peitoral ... Bruno Felipe Mendonça Leitão • Liliam Fernandes de Oliveira • Thiago Torres da Matta

\section{Tratamento Estatístico}

Para aplicação da estatística inferencial, foi realizado a priori o teste de normalidade Shapiro-Wilk. Após a confirmação da normalidade das EM\% de cada sítio, foi aplicada a ANOVA One-Way com medidas repetidas para comparação da variação percentual entre os sítios, caso alguma diferença estatística fosse observada, o post hoc de Bonferroni iria ser usado para identificação. Todo o tratamento estatístico foi realizado pelo pacote comercial GraphPad Prism 5.0 (Graphpad software inc., EUA) com 5\% de significância estatística.

Para avaliação da confiabilidade e variabilidade da medida da EM em cada sítio do PM, foram realizadas duas imagens no mesmo dia para cada voluntário. O coeficiente de correlação intraclasse (ICCr) e 95\% do intervalo de confiança (95\% IC) foram usados para apresentar os dados de confiabilidade e para variabilidade das medidas foi aplicado o coeficiente de variação percentual (CV\%).

\section{Resultados}

Como resultado da confiabilidade e variabilidade da medida, foi encontrado, para o sítio medial, $\mathrm{ICCr}=0,991(\mathrm{p}<0,001), \mathrm{CV} \%$ $=2,97 \%$ e $95 \%$ IC = 0,976-0,997; para o sítio central: $\mathrm{ICCr}=0,983$ $(p<0,001), C V \%=2,24 \%$ e 95\% IC=0,954-0,994; por fim, para o sítio lateral: ICCr =0,971 ( $p<0,001), C V \%=2,70 \%$ e 95\% IC = 0,922-0,989.

A sobrecarga e o número de repetições utilizados nas três séries de supino reto estão expostos em média e desvio-padrão na Tabela 1.

Tabela 1: Média e desvio-padrão das repetições e sobrecargas utilizados na primeira, segunda e terceira série do supino reto.

\begin{tabular}{cccc}
\hline & $1^{\text {a }}$ série & $2^{\text {a }}$ série & $3^{\text {a }}$ série \\
\cline { 2 - 4 } Número de repetições & $13,33 \pm 2,99$ & $8,83 \pm 2,44$ & $8,50 \pm 1,38$ \\
Sobrecarga $(\mathrm{kg})$ & $78,17 \pm 5,75$ & $78,17 \pm 5,75$ & $72,50 \pm 4,36$ \\
\hline
\end{tabular}

Fonte: Elaborada pelos autores, 2018. 
Variação aguda não uniforme da espessura muscular ao longo do peitoral ...

Bruno Felipe Mendonça Leitão • Liliam Fernandes de Oliveira • Thiago Torres da Matta

A média dos valores absolutos da EM dos sítios do PM pré e pós-intervenção estão expostas na Tabela 2.

Tabela 2: Média e desvio-padrão das EM para os três sítios do PM pré e pós.

\begin{tabular}{cccc}
\hline & Medial & Central & Lateral \\
\cline { 2 - 4 } Pré $(\mathrm{cm})$ & $2,33 \pm 0,40$ & $2,97 \pm 0,29$ & $3,47 \pm 0,30$ \\
Pós $(\mathrm{cm})$ & $2,67 \pm 0,44$ & $3,32 \pm 0,36$ & $3,82 \pm 0,39$ \\
\hline
\end{tabular}

Fonte: Elaborada pelos autores, 2018.

Foram encontradas diferenças significativas entre as variações relativas da EM entre os sítios medial $(14,78 \% \pm 5,87)$ e lateral $(10,04 \% \pm 5,20)(p=0,036)(F=4,015)$ (Figura 3). Não foram encontradas diferenças da EM para o sítio central $(11,83 \% \pm 5,99)$ do PM quando comparadas às outras duas medidas.

Figura 3: Valores individuais normalizados da EM expressos em média e desvio-padrão para as medidas realizadas nos três sítios do PM.

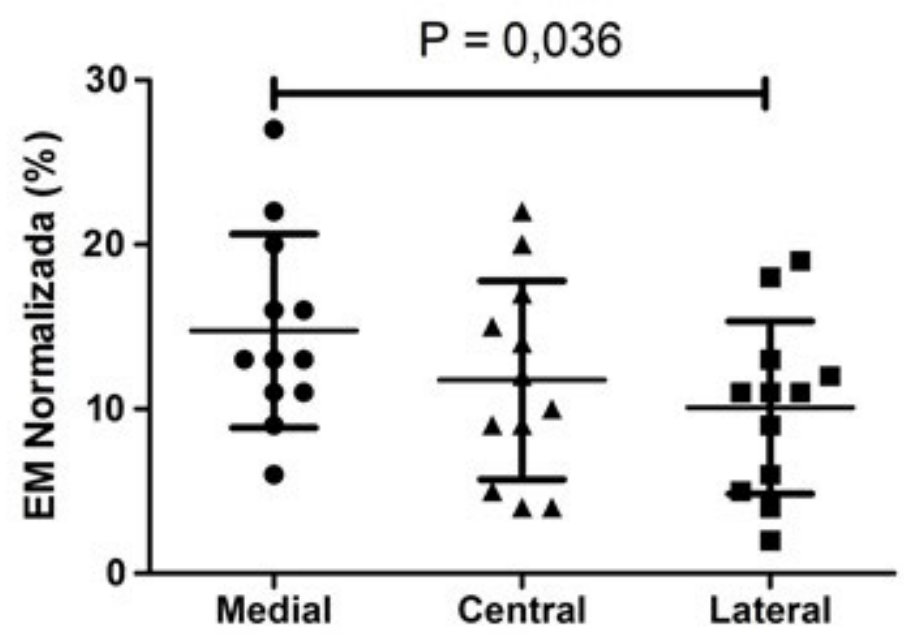

Fonte: Elaborada pelos autores, 2018. 
Variação aguda não uniforme da espessura muscular ao longo do peitoral ... Bruno Felipe Mendonça Leitão • Liliam Fernandes de Oliveira • Thiago Torres da Matta

\section{Discussão}

O objetivo do estudo foi comparar a variação da EM ao longo do PM após uma intervenção aguda de TF em indivíduos treinados, apresentando novos dados para a literatura e promovendo uma base para possível extrapolação nas adaptações crônicas. Como resultado, a variação da EM se mostrou heterogênea, tendo o sítio medial apresentado o maior aumento relativo $(14,78 \%)$ quando comparado ao sítio lateral (10,04\%). Até o presente momento, não muitos estudos haviam analisado o comportamento da EM do PM influenciado pelo TF convencional (ABE et al., 2000; KIKUCHI; NAKAZATO, 2017; OGASAWARA et al., 2012; PRESTES et al., 2017), porém, sem apresentar dados relativos às variações agudas de variáveis da arquitetura muscular entre diferentes comprimentos.

Alguns estudos também mostraram o aumento da EM como efeito agudo de um treinamento. Csapo e colaboradores (2011) reportaram aumento de $7 \%$ da EM a $50 \%$ do vasto lateral após uma série exaustiva de Leg Press unilateral em apenas um sítio muscular. Corroborando esses resultados, Kubo e colaboradores (2001) também apresentaram aumento de aproximadamente 5\% da EM do vasto lateral após diferentes tipos de intervenções para extensores de joelho em $50 \%$ do comprimento da coxa. Esses achados corroboram os dados de Brancaccio e colaboradores (2008), que relataram aumento de aproximadamente $9 \%$ da EM para o quadríceps em $66 \%$ distal do comprimento da coxa, após teste no ciclo-ergômetro até a falha concêntrica. O presente estudo apresentou maior variação da EM (entre 10 e 15\%) quando comparado aos relatos anteriores, possivelmente pelo TF aplicado, o grupamento analisado e a amostra serem diferentes.

Quando comparado aos resultados encontrados por Akagi e colaboradores (2015), que mostraram um aumento de aproximadamente 12 a 14\% da EM em diferentes sítios do tríceps após cinco séries de oito repetições para extensores de cotovelo, as variações percentuais da EM do presente estudo foram semelhantes, 
Variação aguda não uniforme da espessura muscular ao longo do peitoral ...

Bruno Felipe Mendonça Leitão • Liliam Fernandes de Oliveira • Thiago Torres da Matta

provavelmente pelo tipo de intervenção aplicada também ter sido similar com a do presente estudo. Já a hipótese de homogeneidade das variações ao longo do músculo baseada nesse estudo de Akagi e colaboradores (2015), não foi confirmada. Uma das possíveis explicações está relacionada às diferenças na arquitetura dos músculos estudados. Além de não possuir ângulo de penação, o PM apresenta aproximadamente 19,3 cm de comprimento de fibra, enquanto a porção longa do tríceps braquial apresenta apenas 10,2 cm (2011). Brancaccio e colaboradores (2008) atribuíram o aumento da EM aguda à maior perfusão sanguínea, para fornecimento de oxigênio e glicose e remoção de substratos metabólicos da musculatura, assim como a resposta inflamatória promovida pelo aumento de cálcio intracelular. Entretanto, Cleary e colaboradores (2006) afirmaram que essa resposta inflamatória estava associada à dor muscular de início tardio, o que não poderia proporcionar esse aumento da EM de forma aguda.

O aumento da EM condiz com o aumento da perfusão de sangue após uma sessão de TF. Nesse sentido, estudos já associaram esse aumento do fluxo sanguíneo agudo em regiões específicas de um músculo com a ativação muscular (WAKAHARA et al., 2012, 2013, 2017). Nesses estudos, foram apresentadas diferenças de ativação muscular para o reto femoral, entre sítios proximais e distais (mais ativados), em exercício uniarticular (WAKAHARA et al., 2017), e para o tríceps braquial, para exercício uniarticular, os sítios distais apresentaram menor ativação (WAKAHARA et al., 2012) e, para exercício biarticular, os sítios centrais apresentaram maior ativação quando comparados ao proximal (WAKAHARA et al., 2013).

Não foi possível encontrar uma causa direta para os resultados encontrados no presente estudo, entretanto, existem duas possíveis explicações para a maior variação da EM ter sido apresentada no sítio medial. A primeira, baseada nos estudos de Wakahara e colaboradores $(2012,2013,2017)$, na qual os autores associam a ativação de sítios musculares com o redirecionamento do fluxo sanguíneo, é que o sítio medial do PM pode estar sendo mais 
Variação aguda não uniforme da espessura muscular ao longo do peitoral ...

Bruno Felipe Mendonça Leitão • Liliam Fernandes de Oliveira • Thiago Torres da Matta

ativado durante o supino reto, promovendo um maior acúmulo sanguíneo nessa região que pode ser observado através da maior EM\%. Dessa forma, os resultados também podem ser explicados através de um viés cinemático, ou seja, durante a adução horizontal do ombro, para a realização da fase concêntrica do exercício, o trecho do PM próximo ao esterno se mantém menos móvel, enquanto o próximo a articulação glenoumeral se movimenta.

A segunda possível explicação pode estar voltada para a morfologia do músculo, os fascículos da porção lateral do feixe esternal do PM parecem retorcidos antes de se fixar no tendão distal, de forma que seus segmentos inferiores se ligam ao tendão acima dos superiores (CHIAVARAS et al., 2014) e dessa forma dissipando a direção das variações da área de secção transversa de forma que não pode ser observada pela EM. Neste sentido, quando comparado os pontos de inserção do PM, pode-se afirmar que as fibras musculares do trecho lateral são mais compactas gerando menores valores de $\mathrm{EM} \%$, possivelmente, por ter menos espaço para acúmulo sanguíneo do que as fibras do trecho medial.

A Tabela 1 apresenta a média das repetições máximas e das sobrecargas para as três séries de supino reto. A primeira série apresentou uma média de repetições acima da faixa de 8 a 12 RM pretendida, provavelmente, devido ao estímulo verbal dos avaliadores, visto que a sobrecarga adotada para o teste foi de $110 \%$ da sobrecarga utilizada pelos voluntários para 10RM em seus treinos habituais. Entretanto, na segunda e na terceira série, a média de repetições atingiu a faixa estipulada, tendo necessitado de redução de sobrecarga da segunda para terceira série, assim como recomendado por Willardson e colaboradores (2012). Ter apenas questionado os voluntários sobre realizar $1 \mathrm{RM}$ de supino reto com $125 \%$ do peso corporal como sobrecarga é uma limitação do estudo em um dos critérios de caracterização da amostra como treinada. No entanto, a média de sobrecarga relativa a massa corporal dos voluntários nas duas primeiras séries do supino reto foi de $98,94 \%$ e de $91,77 \%$ na última série, auxiliando a caracterização dos voluntários como homens treinados. 
Variação aguda não uniforme da espessura muscular ao longo do peitoral ... Bruno Felipe Mendonça Leitão • Liliam Fernandes de Oliveira • Thiago Torres da Matta

Os mesmos estudos de Wakahara e colaboradores (2012, 2013 , 2017) correlacionaram hipertrofia com a mudança no fluxo sanguíneo regional. Foi verificado que os sítios musculares mais ativados, pelo maior aporte sanguíneo, durante o exercício foram os mais hipertrofiados após 12 semanas de treinamento do mesmo exercício. Desta forma, pode ser que o mesmo ocorra para o PM, tendo o sítio medial mais hipertrofiado, se aplicado um treinamento crônico de supino reto, sendo essa uma das possíveis aplicações práticas do estudo. Uma limitação do presente estudo foi não analisar e comparar a variação da EM no feixe clavicular e nem em diferentes segmentos do feixe esternal do PM, apenas em diferentes sítios do mesmo segmento.

O presente estudo teve como objetivo comparar a variação da EM em diferentes sítios musculares do PM após três séries de supino reto em indivíduos treinados. Neste sentido, observou-se que a EM aumentou com maior magnitude estatística no trecho medial quando comparado ao sítio lateral, evidenciando a variação heterogênea e não confirmando a hipótese. Futuros estudos usando outros exercícios voltados para o PM, como o crucifixo, bem como outras posturas e inclinações do supino, por exemplo, podem ser realizados para evitar generalizações a partir dos achados do presente estudo. Outra questão que ainda deve ser observada em futuros estudos é a avaliação do efeito crônico do supino reto na EM ao longo do PM.

\section{Referências}

$A B E$, T. et al. Time course for strength and muscle thickness changes following upper and lower body resistance training in men and women. European Journal of Applied Physiology and Occupational Physiology, EUA, v. 81, n. 3, p. 0174, 2000. AKAGI, R. et al. Muscle hardness of the triceps brachii before and after a resistance exercise session: A shear wave ultrasound elastography study. Acta Radiologica, EUA, 2015. 
Variação aguda não uniforme da espessura muscular ao longo do peitoral ... Bruno Felipe Mendonça Leitão • Liliam Fernandes de Oliveira • Thiago Torres da Matta

BARNETT, C.; KIPPERS, V.; TURNER, P. Effects of variations of the bench press exercise on the EMG activity of five shoulder muscles. Journal of Strength and Conditioning Research, EUA, v. 9, n. 4, p. 222-227, 1995.

BLAZEVICH, A. J. et al. Training-Specific Muscle Architecture Adaptation after 5-wk Training in Athletes. Medicine and Science in Sports and Exercise, EUA, v. 35, n. 12, p. 2013-2022, 2003.

BRANCACCIO, P. et al. Changes in skeletal muscle architecture following a cycloergometer test to exhaustion in athletes. Journal of Science and Medicine in Sport, EUA, v. 11, n. 6, p. 538-541, 2008.

CHIAVARAS, M. M. et al. Pectoralis major tears: Anatomy, classification, and diagnosis with ultrasound and MR imaging. Skeletal Radiology, EUA, v. 44, n. 2, p. 157-164, 2014.

CLEARY, M. A.; SITLER, M. R.; KENDRICK, Z. V. Dehydration and symptoms of delayed-onset muscle soreness in normothermic men. Journal of Athletic Training, EUA, 2006.

CLIFFORD, P. S. et al. Mechanical compression elicits vasodilatation in rat skeletal muscle feed arteries. Journal of Physiology, EUA, 2006.

CSAPO, R.; ALEGRE, L. M.; BARON, R. Time kinetics of acute changes in muscle architecture in response to resistance exercise. Journal of Science and Medicine in Sport, EUA, v. 14, n. 3, p. 270-274, 2011.

EMA, R. et al. Inhomogeneous architectural changes of the quadriceps femoris induced by resistance training. European Journal of Applied Physiology, EUA, v. 113, n. 11, p. 2691-2703, 2013.

FLAMM, S. D. et al. Redistribution of regional and organ blood volume and effect on cardiac function in relation to upright exercise intensity in healthy human subjects. Circulation, 1990.

FRIDÉN, J.; LIEBER, R. L. Eccentric exercise-induced injuries to contractile and cytoskeletal muscle fibre components. Acta Physiologica Scandinavica. Anais... 2001 
Variação aguda não uniforme da espessura muscular ao longo do peitoral ... Bruno Felipe Mendonça Leitão • Liliam Fernandes de Oliveira • Thiago Torres da Matta

KIKUCHI, N.; NAKAZATO, K. Low-load bench press and push-up induce similar muscle hypertrophy and strength gain. Journal of Exercise Science and Fitness, EUA, v. 15, n. 1, p. 37-42, 2017. $\mathrm{KUBO}, \mathrm{K}$. et al. Influences of repetitive muscle contractions with different modes on tendon elasticity in vivo. J Appl Physiol (1985), EUA, 2001.

LAUVER, J. D.; CAYOT, T. E.; SCHEUERMANN, B. W. Influence of bench angle on upper extremity muscular activation during bench press exercise. European Journal of Sport Science, EUA, v. 16, n. 3, p. 309-316, 2016.

MATTA, T. et al. Strength training's chronic effects on muscle architecture parameters of different arm sites. The Journal of Strength \& Conditioning Research, EUA, 2011.

MATTA, T. T. et al. Heterogeneity of rectus femoris muscle architectural adaptations after two different 14-week resistance training programmes. Clinical Physiology and Functional Imaging, EUA, 2015.

OGASAWARA, R. et al. Time course for arm and chest muscle thickness changes following bench press training. Interventional Medicine and Applied Science, EUA, 2012.

PETERSON, S. L.; RAYAN, G. M. Shoulder and upper arm muscle architecture. Journal of Hand Surgery, EUA, 2011.

PRESTES, J. et al. Strength And Muscular Adaptations Following 6 Weeks Of Rest-Pause Versus Traditional Multiple-Sets Resistance Training In Trained Subjects. Journal of Strength and Conditioning Research, EUA, 2017.

SÁ, M. A. et al. Acute Effects of Different Methods of Stretching and Specific Warm-ups on Muscle Architecture and Strength Performance. Journal of Strength and Conditioning Research, EUA, 2016.

WAKAHARA, T. et al. Association between regional differences in muscle activation in one session of resistance exercise and in 
Variação aguda não uniforme da espessura muscular ao longo do peitoral ... Bruno Felipe Mendonça Leitão • Liliam Fernandes de Oliveira • Thiago Torres da Matta

muscle hypertrophy after resistance training. European Journal of Applied Physiology, EUA, v. 112, n. 4, p. 1569-1576, 2012.

WAKAHARA, T. et al. Nonuniform muscle hypertrophy: Its relation to muscle activation in training session. Medicine and Science in Sports and Exercise, EUA, v. 45, n. 11, p. 2158-2165, 2013.

WAKAHARA, T. et al. Inter- and intramuscular differences in training-induced hypertrophy of the quadriceps femoris: association with muscle activation during the first training session. Clinical Physiology and Functional Imaging, EUA, v. 37, n. 4, p. 405-412, 2017.

WEIR, J. P.; WAGNER, L. L.; HOUSH, T. J. The effect of rest interval length on repeated maximal bench presses. Journal of Strength and Conditioning Research, EUA, 1994.

WILLARDSON, J. M.; SIMAO, R.; FONTANA, F. E. The effect of load reductions on repetition performance for commonly performed multijoint resistance exercises. Journal of Strength and Conditioning Research, EUA, 2012.

\section{Notas}

\section{Financiamento}

A presente pesquisa teve financiamento da FAPERJ (Fundação de Amparo à Pesquisa do Estado do Rio de Janeiro)

\section{Aprovação de comitê de ética em pesquisa}

Pesquisa aprovada pelo Comitê de Ética da Universidade Federal do Rio de Janeiro, título: Análise de parâmetros de textura em ultrassonografias músculo-esqueléticas pós-exercício. 
Variação aguda não uniforme da espessura muscular ao longo do peitoral ...

Bruno Felipe Mendonça Leitão • Liliam Fernandes de Oliveira • Thiago Torres da Matta

\section{Publisher}

Universidade Federal de Goiás. Faculdade de Educação Física e Dança. Publicação no Portal de Periódicos UFG. As ideias expressadas neste artigo são de responsabilidade de seus autores, não representando, necessariamente, a opinião dos editores ou da universidade. 